> Notre étude porte sur la description des propriétés mécaniques des artères carotides et radiales dans une cohorte de 24 patients hémizygotes porteurs de maladie de Fabry authentifiée, comparés à 21 sujets témoins. De plus, nous avons recherché les corrélations entre le remodelage ventriculaire, mesuré à I'IRM et le remodelage artériel. Les paramètres artériels ont été determinés par échotracking vasculaire, technique 10 fois plus précise que les techniques concurrentes. Nous avons trouvé une hypertrophie sévère de la carotide et de l'artère radiale, s'associant à une augmentation de la distensibilité. L'épaississement avec l'âge était exacerbé chez les patients atteints de maladie de Fabry. Le remodelage hypertrophique concentrique ventriculaire était corrélé à la charge hémodynamique et au remodelage artériel. Ces résultats montrent que les tissus cardiovasculaires sont hypersensibles aux signaux trophiques chez les patients atteints de maladie de Fabry. <

\section{Atteinte vasculaire et remodelage ventriculaire gauche dans la maladie de Fabry}

\author{
Pierre Boutouyrie, Elie Mousseaux, \\ Benjamin Cocheteux, Stéphane Laurent, \\ Dominique P. Germain
}

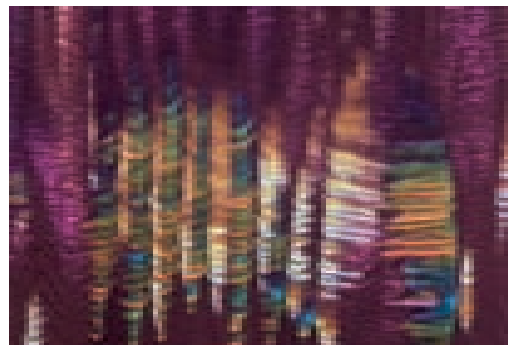

\author{
P. Boutouyrie, S. Laurent : \\ Université Paris-Descartes, \\ Faculté de Médecine, \\ Département de Pharmacologie \\ et Inserm U.652. E. Mousseaux, \\ B. Cocheteux : Département de \\ Pharmacologie, Département \\ de Radiologie. D.P. Germain : \\ Département de Génétique. \\ Hôpital Européen Georges \\ Pompidou, Assistance Publique- \\ Hôpitaux de Paris, Université \\ Paris 5, 20, rue Leblanc, \\ 75015 Paris, France. \\ pierre.boutouyrie@ \\ egp.ap-hop-paris.fr
}

L'atteinte cardiovasculaire est au centre des complications de la maladie de Fabry (MF). En effet, le dépôt de glycosphingolipides se fait préférentiellement dans les artères de petit et moyen calibre, notamment dans l'endothélium, et les principales causes de morbidité et de mortalité précoces sont liées aux complications ischémiques neurologiques, rénales et cardiaques [1-3]. C'est dans ce contexte qu'il s'avérait intéressant de constituer une cohorte de patients atteints de la maladie de Fabry dans l'optique de mieux préciser le phénotype artériel et la pathogénie des lésions vasculaires et cardiaques.

La cohorte des patients suivis dans l'Unité de Génétique Clinique de l'Hôpital Européen Georges Pompidou (HEGP) (Dr D.P. Germain) comporte 97 patients ayant bénéficié de mesures des propriétés géométriques et fonctionnelles artérielles et cardiaques, dans le cadre du bilan réalisé préalablement à la mise sous traitement substitutif par $\alpha$-galactosidase recombinante. Ces patients sont plus souvent de sexe masculin (73), avec un âge moyen de 34 ans (12-79). Ils font partie d'un groupe de plus de
180 patients ayant consulté, dont 45 sont venus plus d'une fois et 12 ont été vus avant et après traitement par l' $\alpha$-galactosidase. Ces patients sont tout à fait typiques quant à leur symptomatologie.

\section{Phénotype artériel de la maladie de Fabry}

Nous nous sommes largement appuyés sur les techniques d'échotracking vasculaire fondées sur l'échographie bidimensionnelle des coupes TM, analyse du signal radiofréquence, qui permet non seulement de mesurer l'épaisseur intima-média et le diamètre avec une précision 10 fois supérieure à la résolution spatiale de l'échographie conventionnelle, mais aussi les mouvements de paroi artérielle avec une résolution nanométrique (Figure 1).

Ces techniques de grande précision sont applicables à tous les axes artériels superficiels. Nous avons choisi deux archétypes: une artère de gros calibre, élastique, l'artère carotide primitive, et une artère de moyen calibre, musculaire, l'artère radiale.

À partir des mesures de diamètre, d'épaisseur et des variations systolo-diastoliques de diamètre, associées 
à la mesure concomitante de la pression locale par tonométrie d'aplanation, nous pouvons calculer la compliance artérielle, en reconstruisant la relation diamètre/pression. La pente de cette relation est la distensibilité artérielle : plus cette pente est importante, plus l'artère est souple, plus cette pente est faible, plus l'artère est rigide.

Nous avons appliqué ces techniques à l'étude d'un groupe de 24 patients mâles hémizygotes $[4,5]$. Notre première constatation fut la présence d'une hypertrophie artérielle considérable intéressant majoritairement les artères de moyen calibre, comme l'artère radiale. Les épaisseurs sont de l'ordre de 400 à $450 \mu \mathrm{m}$ pour les patients atteints de MF, sans superposition avec une population témoin appariée sur l'âge et le sexe.

À un moindre degré, nous retrouvons cette hypertrophie au niveau de l'artère carotide primitive, artère élastique de gros calibre, d'environ $40 \%$, avec toutefois une superposition des valeurs avec celles de certains témoins.

Cette hypertrophie considérable va de pair avec une augmentation de la distensibilité artérielle, notamment pour l'artère radiale. II résulte de cette étude, un phénotype très particulier, spécifique de la MF, associant hypertrophie des artères musculaires et élastiques, avec préservation (voire augmentation) du diamètre interne de l'artère et augmentation de distensibilité des artères musculaires. La rigidité systémique, représentée par la vitesse de l'onde de pouls carotido-fémorale, n'est pas diminuée ( $11,7 \mathrm{~m} / \mathrm{s}$ versus $10,8 \mathrm{~m} / \mathrm{s}$ chez les témoins).

La seconde constatation fut la relation à l'âge des paramètres artériels. L'épaisseur artérielle augmente chez les patients témoins avec l'âge, avec une relation très significative de pente d'environ $2,5 \mu \mathrm{m} / \mathrm{an}$. Quel que soit leur âge, les patients MF ont des valeurs d'épaisseur artérielle beaucoup plus élevées et surtout une pente presque trois fois plus importante en fonction de l'âge, donc une sensibilité particulière au vieillissement.

Une augmentation graduelle et significative de l'épaisseur de l'artère radiale en fonction du nombre d'événements cliniques fut par ailleurs constatée. L'atteinte artérielle est proportionnelle à la sévérité de la maladie. Mais cette évaluation est à prendre avec prudence, car il est difficile de définir un score de sévérité fiable et validé pour cette maladie multisystémique.

Quoi qu'il en soit, il existe un épaississement considérable des artères, notamment au niveau de l'artère radiale - artère musculaire très

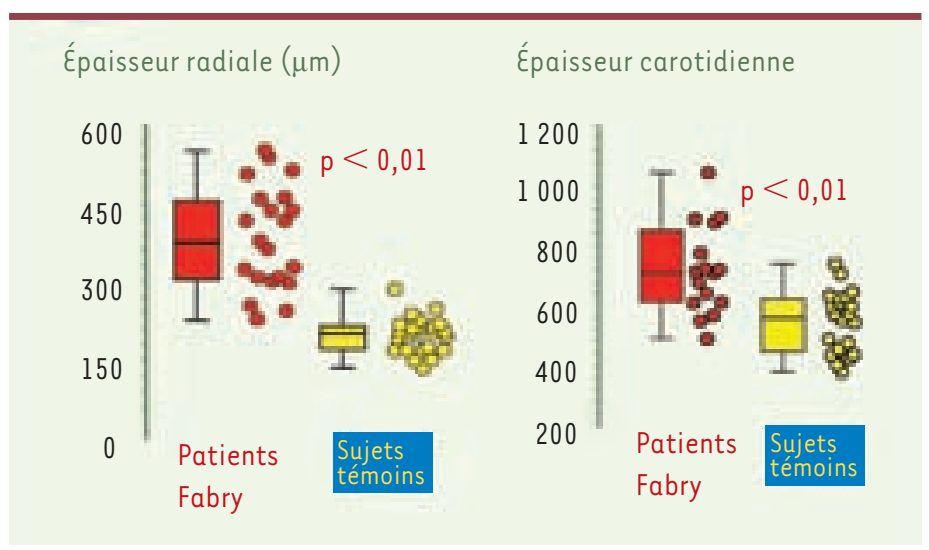

Figure 1. Hypertrophie artérielle chez les patients atteints de maladie de Fabry, comparativement à des sujets témoins appariés sur l'âge et le sexe. dépendante de l'âge - avec une relation à l'âge exacerbée, qui est associé à une augmentation de distensibilité locale, traduisant le fait que le tissu accumulé dans les parois de maladie de Fabry est un tissu souple, riche en éléments cellulaires. L'augmentation de la quantité de tissu compense sa plus grande élasticité, expliquant l'absence de diminution de la rigidité systémique.

\section{Interaction entre rigidité artérielle et remodelage ventriculaire}

Fait déjà signalé par plusieurs auteurs, I'hypertrophie artérielle va bien au-delà de la quantité de Gb3 accumulée dans les parois. La MF est considérée comme une maladie de «surcharge», mais est-ce vraiment l'accumulation de Gb3 dans les cellules musculaires lisses ou dans les cellules endothéliales qui est à elle seule responsable de l'hypertrophie? Les glycosphingolipides représentent moins de $2 \%$ de la masse humide du tissu $[6,7]$, ce qui est insuffisant pour expliquer une augmentation de $200 \%$ de l'épaisseur.

Afin d'expliquer cette constatation expérimentale, nous avons étudié les interactions entre la rigidité artérielle et le remodelage ventriculaire dans la MF afin de tester le rôle de la prolifération dans l'hypertrophie vasculaire et myocardique.

Nous savons que, chez le sujet normotendu et chez l'hypertendu, l'hypertrophie ventriculaire gauche est très dépendante de la charge hémodynamique, et notamment de la rigidité artérielle et de la pression pulsée, qui induisent des hypertrophies concentriques [9-11]. Dans l'hypothèse classique où l'augmentation de masse ventriculaire gauche serait liée au stockage passif de sphingolipides, les conditions de charge hémodynamique ne devraient pas avoir d'influence sur l'hypertrophie. Si au contraire les sphingolipides agissent comme des facteurs de croissance, une exacerbation de la dépendance à la pression de l'hypertrophie ventriculaire gauche devrait apparaître.

Nous avons sélectionné un sous-groupe de 24 patients ayant eu à la fois des mesures artérielles par échotracking vasculaire et une étude de la structure et fonction cardiaque par résonance magnétique nucléaire.

Nos patients avaient une préservation de la fraction d'éjection ventriculaire gauche $(60 \%)$ comme constatée par d'autres auteurs [12, 13], une absence de dilatation ventriculaire gauche, une augmentation assez modeste de la masse ventriculaire gauche, mais surtout un épaississement important des parois $(16,4 \mathrm{~mm}$; normale $<11,5)$, et une épaisseur relative tout à fait considérable $(0,63$; normale $<0,43)$. La majorité $(n=15)$ des patients avaient une hypertrophie concentrique, et 7 patients un 


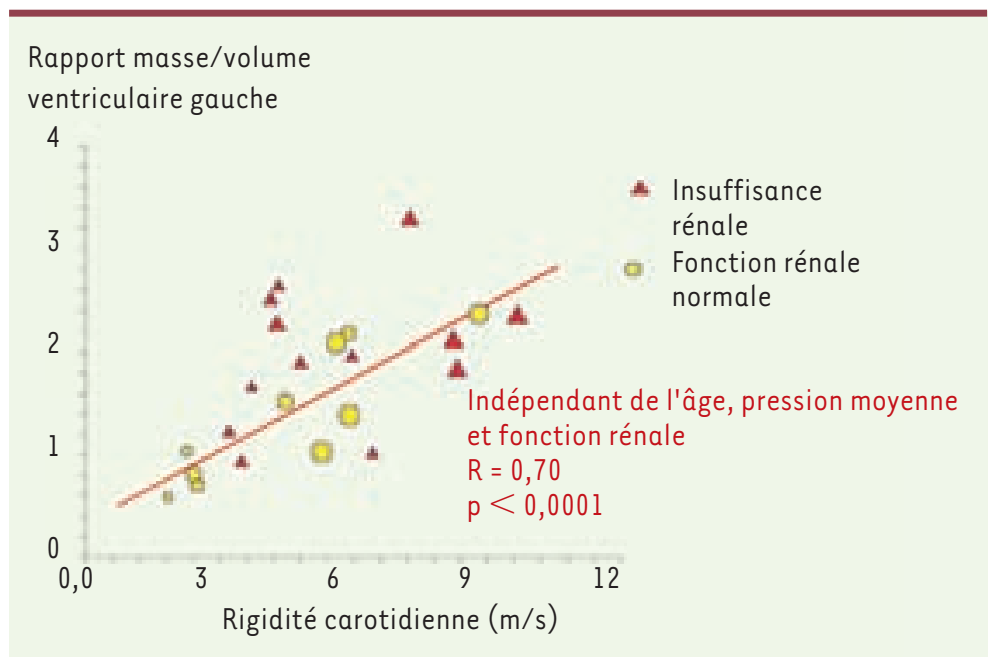

Figure 2. Relation entre remodelage concentrique du ventricule gauche et rigidité carotidienne chez le patient porteur de maladie de Fabry.

remodelage concentrique. Seuls, deux patients, les plus jeunes, avaient une géométrie ventriculaire normale. Notre travail montre donc une corrélation très significative entre la rigidité carotidienne et l'hypertrophie ventriculaire gauche (Figure 2), ici exprimée par le rapport masse/volume télédiastolique (équivalent d'épaisseur relative). Cette relation, indépendante de l'âge, de la pression artérielle et de la fonction rénale est forte, plus pentue que celle retrouvée chez les hypertendus. Elle est identique à la pression pulsée centrale. Elle résulte d'une sensibilité exacerbée du remodelage ventriculaire gauche à la charge hémodynamique. La corrélation de I'index de masse ventriculaire gauche avec un index d'hypertrophie artérielle (moyenne pondérée de l'épaisseur intima-média radiale et carotidienne), est très significative $(r=0,50, p<0,001)$, indépendante de l'âge, de la pression artérielle et de la fonction rénale. Ces résultats indiquent un certain degré de parallélisme entre l'hypertrophie artérielle et l'hypertrophie cardiaque et montrent la sensibilité exacerbée du myocarde aux conditions de charge hémodynamique, renforçant l'hypothèse d'une «accumulation de facteurs de croissance ».

\section{Conclusions}

La maladie de Fabry est caractérisée par une hypertrophie artérielle sévère, dépassant de très loin l'accumulation simple des sphingolipides, prédominant sur les artères de taille moyenne, mais aussi sur les artères de plus grande taille, et associée à une augmentation de la distensibilité. Pour les artères, la diminution de la rigidité du matériau est compensée par l'augmentation de l'épaisseur, sans diminution de la rigidité systémique.
L'hypertrophie ventriculaire gauche est par ailleurs dépendante de la charge hémodynamique qui entretient l'augmentation de la masse cardiaque et du remodelage ventriculaire concentrique. $\diamond$

\section{SUMMARY}

Large artery and left ventricular remodelling in Fabry disease

In the present study, we determined mechanical properties of the carotid and radial arteries in a cohort of 24 hemizygous male patients with biologically proven Fabry disease (FD), compared with 21 age and sex matched normal controls. We further sought for correlations between left ventricle geometry mesured by MRI, and arterial remodelling. Arterial parameters were obtained with a high precision echotracking device, 10 time more precise than concurrent techniques. We found a severe hypertrophy associated with increased distensibility, both for the carotid and the radial artery. Thickening with age was exacerbated in FD patients. Left ventricule remodelling was dependent on hemodynamic load and proportional to arterial hypertrophy. These results show that tissues of the cardiovascular system tend to be oversensitive to trophic signals in patients with Fabry disease. $\diamond$

\section{RÉFÉRENCES}

1. MacDermot KD, Holmes A, Miners AH. Anderson-Fabry disease : clinical manifestations and impact of disease in a cohort of 60 obligate carrier females. J Med Genet 2001 ; 38: 769-75.

2. MacDermot KD, Holmes A, Miners AH. Anderson-Fabry disease : clinical manifestations and impact of disease in a cohort of 98 hemizygous males. J Med Genet 2001 ; 38:750-60.

3. Germain DP. Fabry's disease (alpha-galactosidase-A deficiency) : physiopathology, clinical signs, and genetic aspects. J Soc Biol $2002 ; 196: 161-73$.

4. Boutouyrie $P$, Laurent $S$, Laloux B, et al. Arterial remodelling in Fabry disease. Acta Paediatr $2002 ; 91$ (suppl) : 62-6.

5. Boutouyrie $P$, Laurent $S$, Laloux $B$, et al. Non-invasive evaluation of arterial involvement in patients affected with Fabry disease. J Med Genet $2001 ; 38: 629-31$.

6. Elleder M, Bradova V, Smid F, et al. Cardiocyte storage and hypertrophy as a sole manifestation of Fabry's disease. Report on a case simulating hypertrophic non-obstructive cardiomyopathy. Virchows Arch A Pathol Anat Histopathol $1990 ; 417$ : 449-55.

7. Desnick RJ, Blieden LC, Sharp HL, et al. Cardiac valvular anomalies in Fabry disease. Clinical, morphologic, and biochemical studies. Circulation 1976; 54:818-25.

8. Chatterjee S. Sphingolipids in atherosclerosis and vascular biology. Arterioscler Thromb Vasc Biol 1998; 18 : 1523-33.

9. Saba PS, Roman MJ, Pini R, et al. Relation of arterial pressure waveform to left ventricular and carotid anatomy in normotensive subjects. J Am Coll Cardiol 1993; 22 : 1873-80.

10. Ganau A, Devereux RB, Roman MJ, et al. Patterns of left ventricular hypertrophy and geometric remodeling in essential hypertension. J Am Coll Cardiol 1992; 19: 1550-8.

11. Boutouyrie $P$, Laurent $S$, Girerd $X$, et al. Common carotid artery stiffness and patterns of left ventricular hypertrophy in hypertensive patients. Hypertension $1995 ; 25: 651-9$.

12. Fisher $\varepsilon A$, Desnick RJ, Gordon RE, et al. Fabry disease : an unusual cause of severe coronary disease in a young man. Ann Intern Med $1992 ; 117: 221-3$.

13. Linhart A, Palecek T, Bultas J, et al. New insights in cardiac structural changes in patients with Fabry's disease. Am Heart J $2000 ; 139$ : 1101-8.

\section{TIRÉS À PART}

P. Boutouyrie 